Copyright by the American Institute of Physics (AIP). Yan, Yongke; Zhou, Yuan; Priya, Shashank, "Giant self-biased

magnetoelectric coupling in co-fired textured layered composites," Appl. Phys. Lett. 102, 052907 (2013); http:// dx.doi.org/10.1063/1.4791685

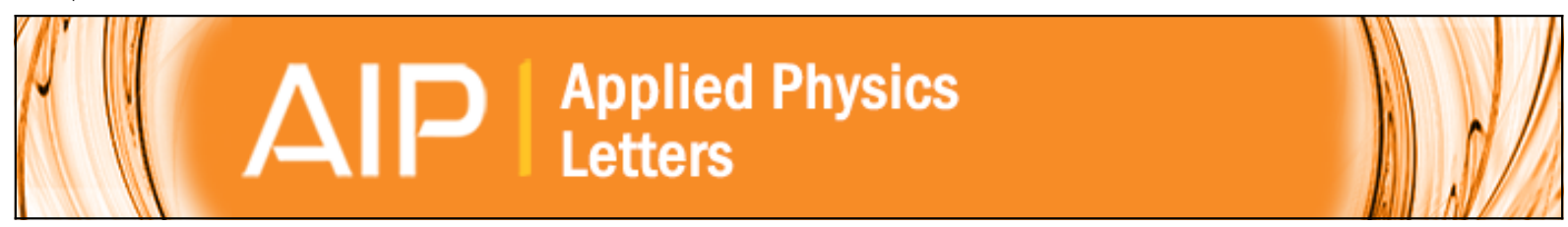

Giant self-biased magnetoelectric coupling in co-fired textured layered composites

Yongke Yan, Yuan Zhou, and Shashank Priya

Citation: Applied Physics Letters 102, 052907 (2013); doi: 10.1063/1.4791685

View online: http://dx.doi.org/10.1063/1.4791685

View Table of Contents: http://scitation.aip.org/content/aip/journal/apl/102/5?ver=pdfcov

Published by the AIP Publishing

$\underset{\substack{\text { Aubloning } \\ \text { Alp }}}{A}$ Re-register for Table of Content Alerts

Create a profile.

Sign up today! 


\title{
Giant self-biased magnetoelectric coupling in co-fired textured layered composites
}

\author{
Yongke Yan, ${ }^{\text {a) }}$ Yuan Zhou, and Shashank Priya ${ }^{\text {b) }}$ \\ Bio-Inspired Materials and Devices Laboratory (BMDL), Center for Energy Harvesting Materials \\ and Systems (CEHMS), Virginia Tech, Virginia 24061, USA
}

(Received 6 November 2012; accepted 24 January 2013; published online 8 February 2013)

\begin{abstract}
Co-fired magnetostrictive/piezoelectric/magnetostrictive laminate structure with silver inner electrode was synthesized and characterized. We demonstrate integration of textured piezoelectric microstructure with the cost-effective low-temperature co-fired layered structure to achieve strong magnetoelectric coupling. Using the co-fired composite, a strategy was developed based upon the hysteretic response of nickel-copper-zinc ferrite magnetostrictive materials to achieve peak magnetoelectric response at zero DC bias, referred as self-biased magnetoelectric response. Fundamental understanding of self-bias phenomenon in composites with single phase magnetic material was investigated by quantifying the magnetization and piezomagnetic changes with applied DC field. We delineate the contribution arising from the interfacial strain and inherent magnetic hysteretic behavior of copper modified nickel-zinc ferrite towards self-bias response. (C) 2013 American Institute of Physics. [http://dx.doi.org/10.1063/1.4791685]
\end{abstract}

The magnetoelectric (ME) effect is defined as the change in dielectric polarization $(P)$ of a material under an applied magnetic field $(H)$ or an induced magnetization $(M)$ under an external electric field $(E) .{ }^{1,2}$ ME coupling of magnetic and electric order parameters can be used in various applications such as magnetic field sensors, transducers, filters, phase shifters, and magnetic switches. ${ }^{3}$ It has been shown both experimentally and theoretically that the strainmediated ME coupling in magnetostrictive-piezoelectric layered composite or laminate is several orders of magnitude larger as compared to single phase materials. ${ }^{3,4}$ Giant magnetoelectric (GME) effect has been reported in a variety of magnetostrictive-piezoelectric laminate composites consisting of ferrite, Terfenol-D, and Metglas as magnetostrictive phase and $\mathrm{Pb}(\mathrm{Zr}, \mathrm{Ti}) \mathrm{O}_{3}(\mathrm{PZT})$ and $\mathrm{Pb}\left(\mathrm{Mg}_{1 / 3} \mathrm{Nb}_{2 / 3}\right) \mathrm{O}_{3}-\mathrm{PbTiO}_{3}$ (PMN-PT) as piezoelectric phase. ${ }^{3-8}$ In these composites, the measured ME coefficient was found to be directly related to the effectiveness of elastic coupling at the interface of two phases.

Figure 1 schematically depicts the four types of layered ME composite structure (type A-D) synthesized by different processing techniques. The widely used method for synthesis of laminate composites is bonding of magnetostrictive layer (such as Metglas, Terfenol-D) and piezoelectric layer (such as PZT, PMN-PT) using epoxy resin (Fig. 1(a)). This method limits the misfit strain at the interface arising due to thermal expansion mismatch between the layers and atomic interdiffusion and/or chemical reaction between the layers. However, the epoxy layer is much softer than both magnetostrictive alloy and ferroelectric ceramic, and thus it will dampen the generated strain resulting in loss of efficiency. Metglas/piezofiber push-pull structure as shown in Fig. 1(b) was developed to reduce the structural clamping and utilize longitudinal operational mode $\left(d_{33}>2 d_{31}\right.$ since Poisson's

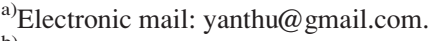

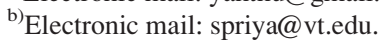

ratio is smaller than 0.5). In this structure, an inter-digitized (ID) electrode pattern was attached on both surfaces of the piezo-fiber $\left(40 \times 2 \times 0.2 \mathrm{~mm}^{3}\right)$ layer using epoxy resin. The ME voltage coefficient $\left(\alpha_{M E}\right)$ of this type-B structure is nearly an order of magnitude higher than those of type-A ME laminates (Fig. 1(a)). ${ }^{5,6}$ However, the epoxy layer also limits the ME response due to low mechanical strength and contributes towards the noise floor and aging. ${ }^{9}$ Furthermore, high cost of raw materials (large dimension PMN-PT single crystal fibers) and manual fabrication process (polishing on the order of $200 \mu \mathrm{m}$ followed by lamination) presents challenge in implementation at commercial scale.

In contrast to bonding, co-firing of layered ME composite provides compatibility with current industrial production process commonly used for fabrication of multilayer capacitors (MLCs). Recently, ME sensor based upon $\mathrm{Ni}-\mathrm{BaTiO}_{3}$ MLCs was reported which laid the foundation for future (a)

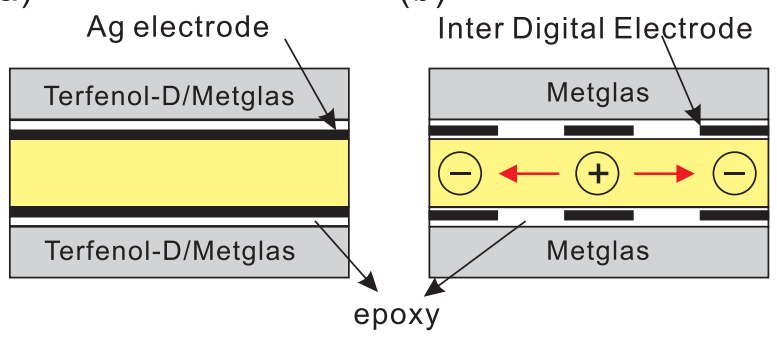

(c)

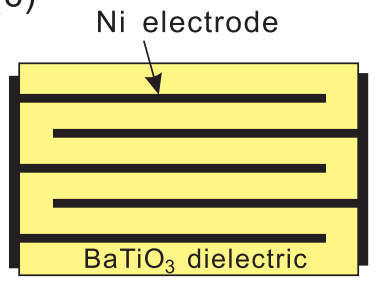

(d)

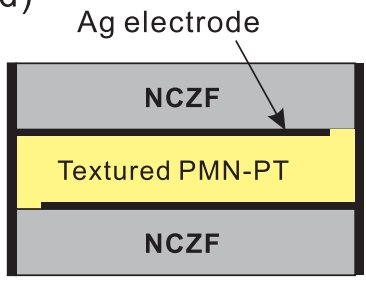

FIG. 1. Schematic diagrams of different types of layered ME composite structures. 
commercialization of this technology. ${ }^{10}$ As shown in Fig. $1(\mathrm{c})$, this type-C structure consists of alternate $\mathrm{BaTiO}_{3}$ thin film layers and ferromagnetic $\mathrm{Ni}$ internal electrodes. The magnitude of $\alpha_{E}$ was found to be $7.1 \mathrm{mV} \mathrm{cm}^{-1} \mathrm{Oe}^{-1}$ which is extremely low, but the projected overall cost was also extremely low. In order to increase the ME effect while keeping the cost down, materials with large magnetostrictive and piezoelectric coefficient are needed in the co-fired configuration. Srinivasan et al. have reported the performance of PZT/ $\mathrm{NiFe}_{2} \mathrm{O}_{4}$ layered composites synthesized by tape casting and co-firing process. ${ }^{11} \mathrm{~A}$ high $\alpha_{E}$ of $400 \mathrm{mV} \mathrm{cm}^{-1} \mathrm{Oe}^{-1}$ was found for the PZT/NiFe $\mathrm{O}_{4}$ multilayer stacks (2 to 21 layers). However, high co-firing temperature (1400-1500 K) and no inner electrode limited the poling of piezoelectric materials, and the process was not compatible with low-temperature cofired ceramics (LTCC) process required for capacitors, inductors, resistors, transformers, and hybrid circuits.

Besides the consideration of the effective strain coupling between magnetostrictive phase and piezoelectric phase, the ME coefficient is strongly dependent upon the intrinsic properties of each phase. For 2-2 type laminate composites, the transverse ME coefficient $\left(\alpha_{\mathrm{E}, 31}\right)$ is given as ${ }^{12}$

$$
\alpha_{E, 31}=\frac{E_{3}}{H_{1}}=\frac{-v(1-v)\left({ }^{m} q_{11}+{ }^{m} q_{21}\right)^{p} d_{31}}{{ }_{\varepsilon_{33}\left({ }^{m} s_{12}+{ }^{m} s_{11}\right) v+{ }^{p} \varepsilon_{33}\left({ }^{p} s_{11}+{ }^{p} s_{12}\right)(1-v)-2^{p} d_{31}^{2}(1-v)}},
$$

where $E_{k}$ and $H_{k}$ are vector components of the electric and magnetic field, $s_{i j}$ is an compliance coefficient, $d_{k i}$ is a piezoelectric coefficient, $q_{k i}$ is a piezomagnetic coefficient, and $\varepsilon_{k n}$ is an permittivity. The superscripts " $m$ " and " $p$ " represent the magnetostrictive and piezoelectric phase, respectively. This relationship clearly reflects the important role of piezomagnetic coefficient towards achieving the large magnetoelectric response. At the same time, it also shows the fundamental limitation of the piezoelectric material. For high $\alpha_{\mathrm{E}}$, one would require high $d_{\mathrm{ki}}$ and low $\varepsilon_{k n}$, but in most of the cases both these coefficients vary in the same direction. Here, we demonstrate template grain growth (TGG) technique that yields suitable microstructure in order to overcome these material challenges. ${ }^{13-18}$ Using this technique, we achieved two goals: (1) $\langle 001\rangle$ texturing (grain orientation along the $\langle 001\rangle$ crystal- lographic direction) of piezoelectric ceramic with engineered domain state similar to that of $\langle 001\rangle$ single crystals to obtain high $d$ values, and (2) suppression of $\varepsilon$ of textured piezoelectric ceramic through the use of low $\varepsilon$ template seeds.

We investigated a co-fired magnetostrictive/piezoelectric/magnetostrictive $(\mathrm{M} / \mathrm{P} / \mathrm{M})$ laminate structure with $\mathrm{Ag}$ inner electrode as shown in Fig. 1(d). Compositions corresponding to $\mathrm{Pb}\left(\mathrm{Mg}_{1 / 3} \mathrm{Nb}_{2 / 3}\right) \mathrm{O}_{3}-32.5 \mathrm{PbTiO}_{3}$ [PMN-PT] and $\left(\mathrm{Ni}_{0.6} \mathrm{Cu}_{0.2} \mathrm{Zn}_{0.2}\right) \mathrm{Fe}_{2} \mathrm{O}_{4}$ [NCZF] were used as piezoelectric and magnetostrictive materials, respectively. Low relative permittivity $\langle 001\rangle$ BT micro-crystals $\left(\varepsilon_{\mathrm{r}}=130\right)$ were selected as templates. Experimental details can be found in Refs. 19 and 20.

Figure 2(a) shows the optical image of co-fired NCZF/ textured-PMN-PT/NCZF (abbreviated as C-N/T/N) laminate
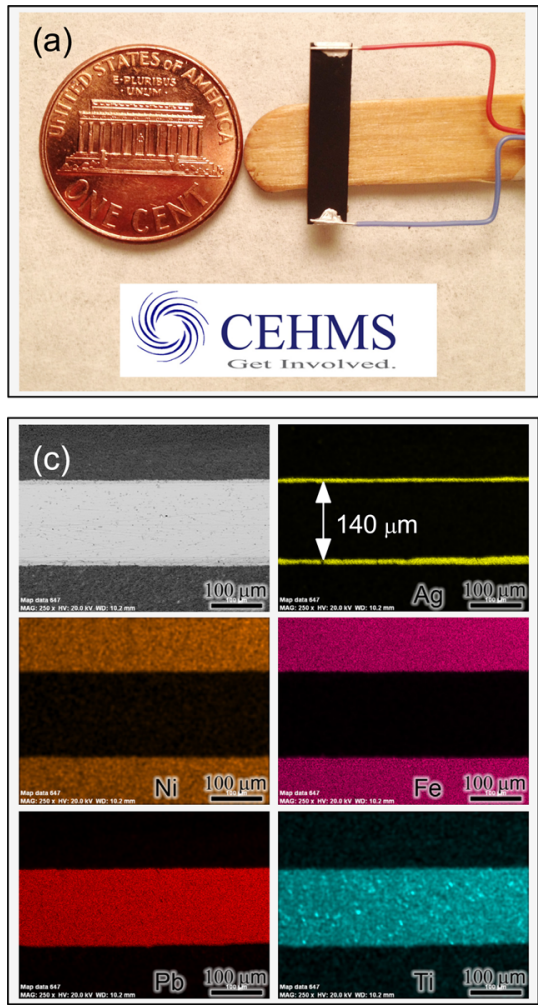
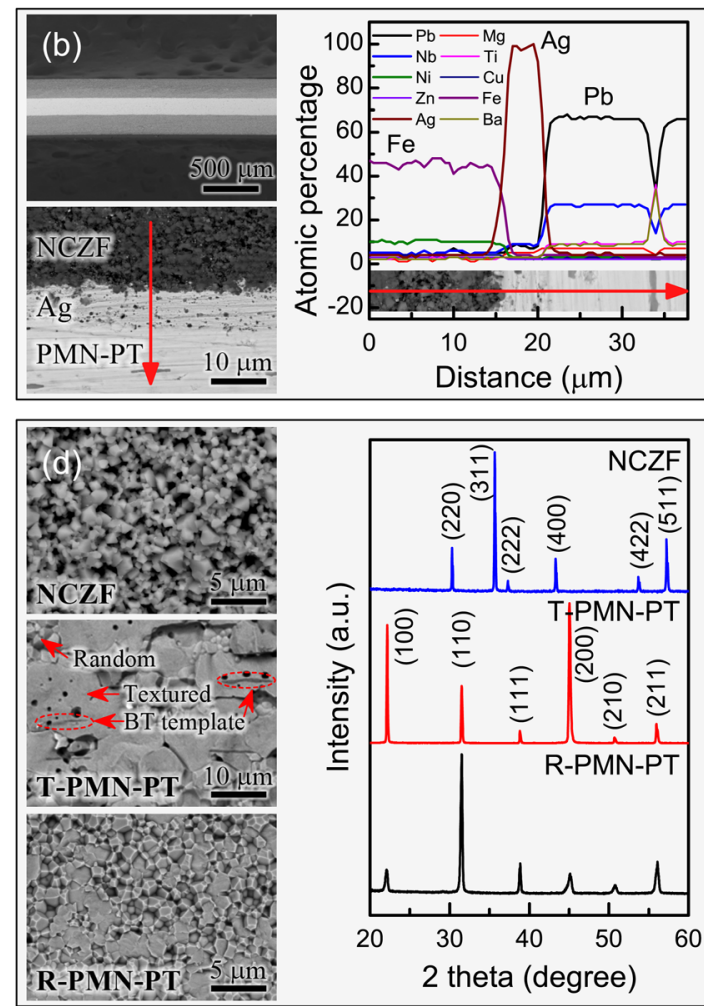

FIG. 2. (a) Optical image of co-fired NCZF/T-PMN-PT/NCZF laminate; (b) polished cross-sectional SEM images and EDS/SEM element line scanning analysis; (c) element mapping of cofired NCZF/T-PMN-PT/NCZF laminate; (d) fracture cross-sectional SEM images and planar XRD patterns of different layers. 
composite synthesized at $930{ }^{\circ} \mathrm{C}$ for $4 \mathrm{~h}$ with dimensions $16 \times 4 \times 0.4 \mathrm{~mm}^{3}$. Co-firing of piezoelectric ceramic layer, Ag metal electrode layer, and ferrite ceramic layer is extremely challenging due to the large difference in shrinkage rates and thermal expansion mismatch. By using fine PMN-PT matrix powder and by adjusting the ratio of sintering aid $(\mathrm{Cu})$ content in NZF as described in the experimental section, we successfully co-fired the multilayer composite. Fig. 2(b) shows the cross-sectional scanning electron microscopy (SEM) images of C-N/T/N composite. There was no trace of delamination or micro-crack and the thickness of textured PMN-PT piezoelectric layer, and NCZF magnetoelectric layer was similar. The interfacial atomic diffusion and/or chemical reactions occurring during the sintering process at high temperature were found to be negligible. Energy dispersive spectroscopy (EDS) line scanning analysis results are shown in Fig. 2(b) indicating the high chemical stability of NCZF, Ag, and PMN-PT. In a prior study conducted on NCZF/PZN-PZT system, the diffusion length for $\mathrm{Cu}$ was found to be in the range of $30 \mu \mathrm{m} .{ }^{21}$ In our case, Ag electrode layer acts as an effective barrier layer for $\mathrm{Cu}$ migration and thus provides a sharp interface by limiting the diffusion. EDS analysis of C-N/T/N cross-sectional area (Fig. 2(c)) shows the dense microstructure and sharp interface across different layers. The thickness of Ag electrode and PMN-PT piezoelectric layer was $10 \mu \mathrm{m}$ and $140 \mu \mathrm{m}$, respectively. Fig. 2(d) shows the fracture surface SEM images and corresponding planar x-ray diffraction (XRD) patterns of different layers. For textured PMN-PT layer synthesized by TGG method, brick wall-like microstructure with well aligned BT templates (black lines) in the PMN-PT matrix was obtained. Increase of $\{001\}$ XRD peak intensity and decrease of other peaks clearly indicates the formation of texture. In TGG process, BT template crystals are aligned in PMN-PT matrix powder by tape-casting process. High temperature treatment (or sintering process) results in the nucleation and growth of PMN-PT matrix on the aligned BT template crystals yielding textured ceramics. At the co-firing temperature of $930^{\circ} \mathrm{C}$, $66 \%$ texture degree was obtained.

Figure 3(a) shows the change in ME voltage coefficient $\left(\alpha_{E}\right)$ as a function of DC magnetic field at off-resonance frequency of $1 \mathrm{kHz}$. Although the magnetostriction of Metglas $(\lambda=40 \mathrm{ppm})$ is twice that of NCZF $(\lambda \approx 20 \mathrm{ppm})$, the maximum $\alpha_{E}$ of co-fired NCZF/random-PMN-PT/NCZF (abbreviated as $\mathrm{C}-\mathrm{N} / \mathrm{R} / \mathrm{N}$ ) is two times as that of epoxy bonded Metglas/random-PMN-PT/Metglas (abbreviated as B-M/R/ M) laminate. Further improvement was achieved for co-fired textured sample $(\mathrm{C}-\mathrm{N} / \mathrm{T} / \mathrm{N})$. The large enhancement in $\langle 001\rangle$ textured ceramics was related to the formation of "engineered domain state" which facilitates the rotation of $\langle 111\rangle$ polarization toward the $\langle 001\rangle$ direction. ${ }^{22,23}$ In Fig. $3(\mathrm{a})$, it can also be observed that there is a strong hysteretic $\mathrm{ME}$ response in the co-fired composite. Considering the relation $\alpha \propto q=d \lambda / d H_{\mathrm{DC}}$, we estimated the effective $\lambda$ behavior by integrating $\alpha$ with respect to the $H_{\mathrm{DC}}$ as shown in Fig. 3(b). The epoxy bonded Metglas/PMN-PT/Metglas shows symmetrical $\lambda$-behavior with respect to the $H_{\mathrm{DC}}$, while the tendency of $\lambda$ for co-fired composites was found to be asymmetric. The variation of $\alpha_{E}$ with frequency of applied $H_{\mathrm{AC}}$ is shown in Fig. 3(c). It can be seen that the maximum $\alpha_{E}$ displays excellent stability. A giant $\mathrm{ME}$ voltage coefficient $\left(>1200 \mathrm{mV} \mathrm{cm}^{-1} \mathrm{Oe}^{-1}\right)$ at zero-bias was achieved in C-N/T/ $\mathrm{N}$ composite. Figure 3(d) shows the dynamic change in charge $(Q)$ or polarization $(P)$ of ME composite (Radiant: Precision Premier II, USA) measured under the condition of $H_{\mathrm{AC}}=1 \mathrm{Oe}$ at $f=1 \mathrm{kHz}$ and $H_{\mathrm{DC}}=0$ Oe. ME charge coefficient $\alpha_{M E, Q}$ can be written as

$$
\alpha_{M E, Q}=\frac{d E}{d H}=\frac{d Q}{d H_{A C}} \times \frac{1}{C t}=\frac{d P}{d H_{A C}} \times \frac{A}{C t},
$$
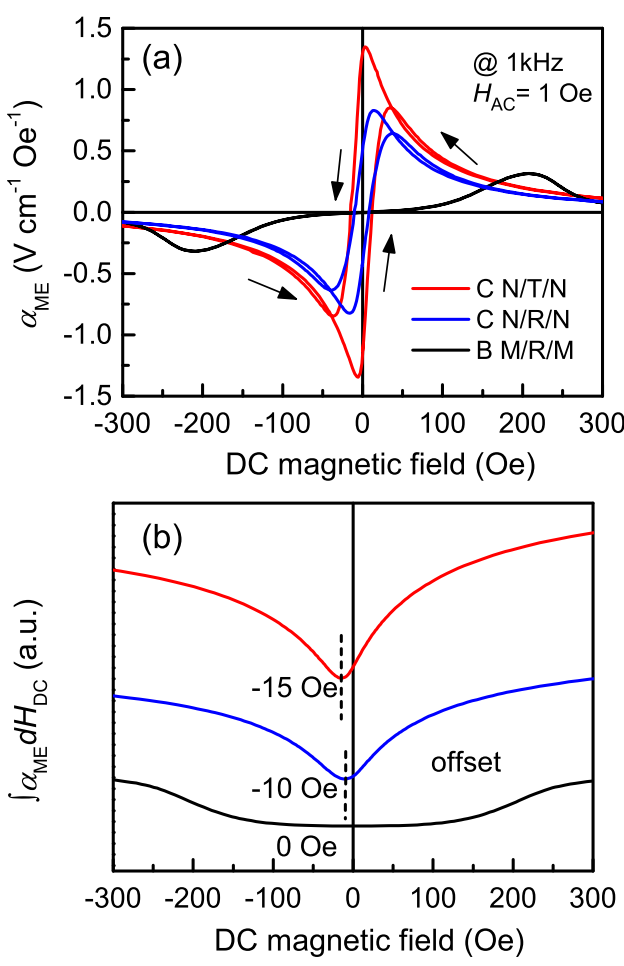
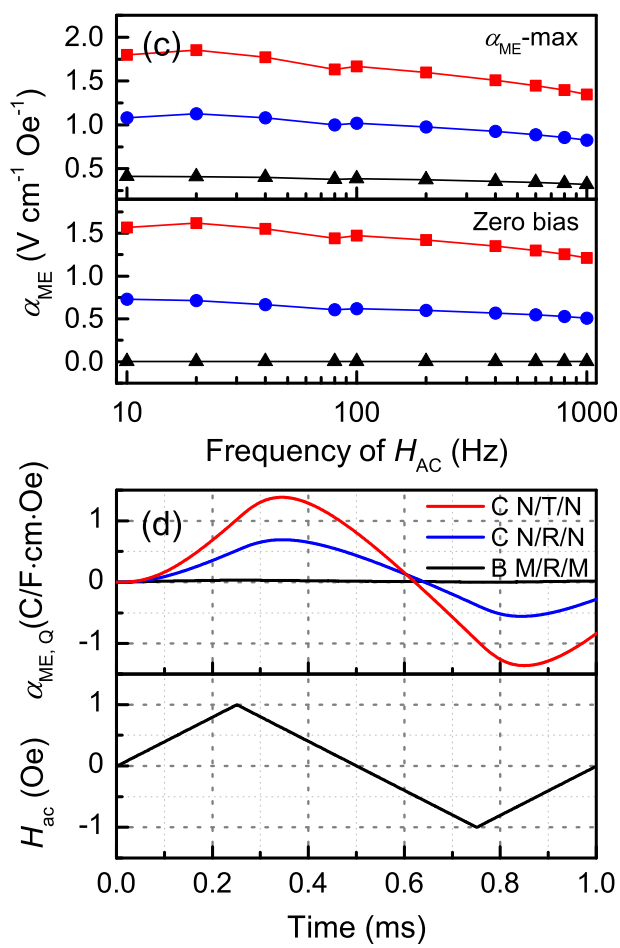

FIG. 3. (a) ME voltage coefficients $\left(\alpha_{M E}\right)$ of co-fired NCZF/T-PMN-PT/ NCZF (C-N/T/N), NCZF/R-PMN-PT/ NCZF $(\mathrm{C}-\mathrm{N} / \mathrm{R} / \mathrm{N})$, and epoxy bonded Metglas/R-PMN-PT/Metglas (B-M/R/ M) laminate. (b) Integral values of $\alpha_{M E}$ with respect to the DC magnetic field. (c) ME voltage coefficients $\left(\alpha_{M E}\right)$ as a function of the frequency of applied ac magnetic field $\left(H_{A C}\right)$. (d) Dynamic ME charge coefficients $\left(\alpha_{M E}\right)$ and applied ac magnetic field spectra as a function of time for C-N/T/N, C-N/R/N composite, and epoxy bonded Metglas/R-PMN-PT/ Metglas (B-M/R/M) laminate, under zero-DC magnetic field. 


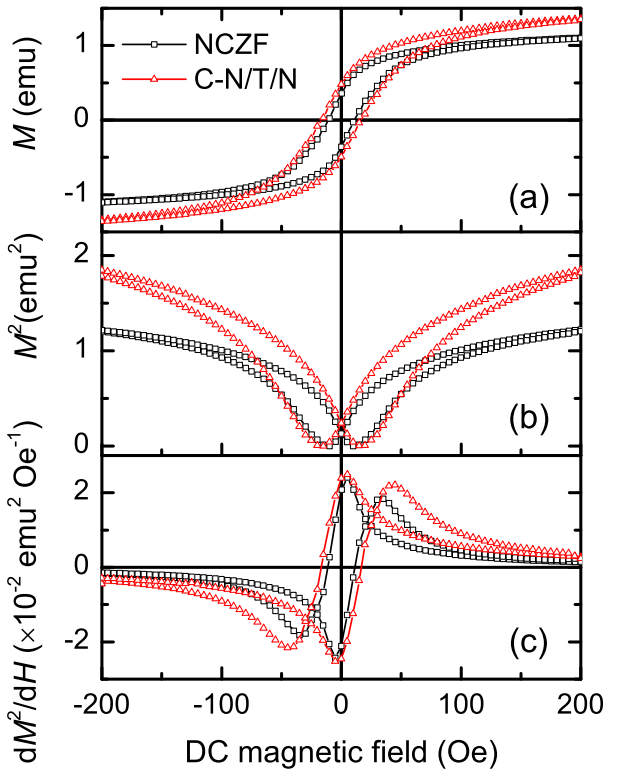

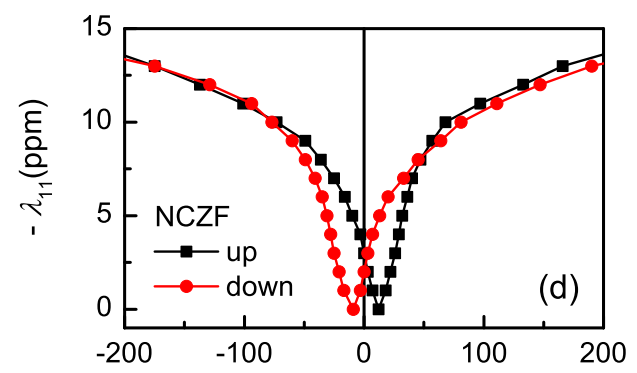

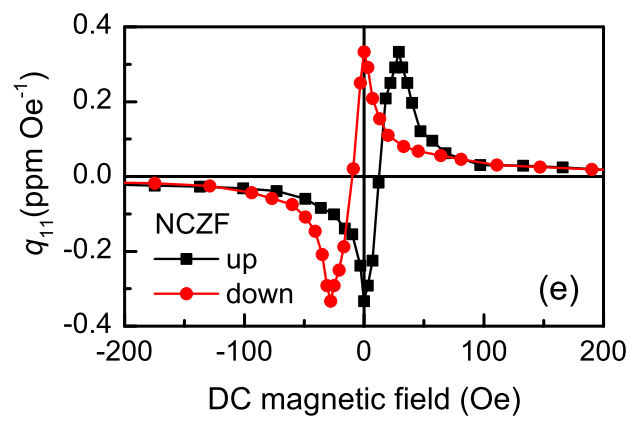

FIG. 4. (a) Magnetization-magnetic field $(M-H)$ loops, (b) square of magnetization as a function of magnetic field, and (c) differential of square magnetizationmagnetic field $\left[\left(\mathrm{d} M^{2} / \mathrm{d} H\right)-H\right]$ plot for pure NCZF (not co-fired) and C-N/T/N structure. DC magnetic field $\left(H_{\mathrm{DC}}\right)$ dependence of (d) the magnetostriction $(\lambda)$ and (e) piezomagnetic coefficients $(q)$ of NCZF (not co-fired). where $C, A$, and $t$ are the capacitance, area, and thickness of the piezoelectric layer, respectively. Large polarization and charge induced by AC magnetic field under zero DC bias and calculated ME charge coefficient $\alpha_{M E, Q}$ is shown in Fig. 3 (d) further confirming the presence of strong ME effect under zero bias. The time delay of $\alpha_{M E, Q}$ related to $H_{\mathrm{AC}}$ can be observed in Fig. 3(d), which is attributed to the interface between magnetostrictive and piezoelectric layer. Compared to the ME coefficient of $30 \mathrm{mV} \mathrm{cm} \mathrm{cm}^{-1} \mathrm{Oe}^{-1}$ obtained for NKNLS-NZF/Ni/NKNLS-NZF trilayer laminate $(40 \times)$, $\sim 100 \mathrm{mV} \mathrm{cm}^{-1} \mathrm{Oe}^{-1}$ obtained for Ni-PZN-PZT bilayer laminate $(12 \times)$, and $\sim 400 \mathrm{mV} \mathrm{cm}{ }^{-1} \mathrm{Oe}^{-1}$ obtained for functionally graded Ni-NZFO-PZT laminate composites $(3 \times)$ at zero-bias, our co-fired composite exhibited extremely high response. ${ }^{24-26}$ Recently, slightly larger zero-bias magnetoelectric coefficient of $1.65 \mathrm{~V} \mathrm{~cm} \mathrm{~cm}^{-1} \mathrm{Oe}^{-1}$ in a quasi-one dimensional ME sensor has been reported at $100 \mathrm{~Hz}$ compared to our case $\left(1.47 \mathrm{~V} \mathrm{~cm}^{-1} \mathrm{Oe}^{-1}\right) .{ }^{27}$ However, our cofired multilayer composite based on LTCC has advantage of integration with other circuit components such as multilayer capacitor, resistor, inductor, and conductor to fabricate fully packaged electronic devices.

The giant ME response of co-fired composite and selfbiased hysteretic behavior can be explained by taking into account the piezoelectric and magnetostrictive characteristics. Figure of merit for the ME composite can be derived as following:

$$
\alpha_{M E}=\frac{\partial E}{\partial H}=\underbrace{\left|\frac{\partial E}{\partial D} \times \frac{\partial D}{\partial T} \times \frac{\partial T}{\partial S}\right|}_{P \text {-layer }} \times \underbrace{\left|\frac{\partial S}{\partial H}\right|}_{P \text {-layer }}=\left|\frac{d}{\varepsilon \times s}\right| \times q,
$$

where $E$ is the output electric field, $H$ is the applied magnetic field, $S$ is the mechanical strain, $T$ is the mechanical stress, $D$ is the electric displacement, $d$ is the piezoelectric constant, $\varepsilon$ is the dielectric constant, $s$ is the elastic compliance of piezoelectric layer ( $P$-layer), and $q$ is piezomagnetic coefficient of magnetostriction layer (M-layer). Since the parameters $d, \varepsilon$, and $s$ of piezoelectric materials are independent of applied magnetic field $(H)$, we focused our investigation on the magnetization $(M)$ of NCZF as a function of magnetic field in longitudinal direction by using vibrating sample magnetometer (VSM) as shown in Fig. 4(a). The ME behavior can be correlated with magnetostriction $(\lambda)$ and magnetization $(M)$ as $^{28}$

$$
\varphi \sim \frac{3 \lambda \sigma}{\left(K+2 \pi M^{2}\right)} \text { or } \lambda \propto M^{2},
$$

where $\varphi$ is the angle of magnetic moments, $K$ and $\sigma$ are the anisotropy constant and stress, respectively. As shown in Figs. 4(b) and 4(c), $M^{2}-H$ and $\partial M^{2} / \partial H-H$ curves can be used to predict the nature of the magnetostriction coefficient ( $\lambda$ ) and piezomagnetic coefficient $(q=d \lambda / d H)$. To further confirm the variation of $q$ as a function of $H_{\mathrm{DC}}$, in-plane magnetostriction coefficient $\left(\lambda_{11}\right)$ was measured in parallel to applied $H_{\mathrm{DC}}$ by strain gauge method (Fig. 4(d)). As shown in Fig. 4(e), the behavior of in-plane piezomagnetic coefficient $\left(q_{\mathrm{ij}}=d \lambda_{\mathrm{ij}} / \mathrm{d} H\right)$ was similar to that of $\partial M^{2} / \partial H$. Due to the inherent hysteresis in NCZF, the magnetostriction coefficient $(\lambda)$ is asymmetric and displays "butterfly" like characteristic. The data in Fig. 4 confirm that the self-bias effect is mainly related to the hysteretic response of ferrite layer.

Table I lists the dielectric and ME properties of co-fired composites. Following conclusion can be drawn from this data: (1) Compared to epoxy bonded Metglas/PMN-PT/Metglas, the maximum $\alpha_{E}$ of NCZF/R-PMN-PT/NCZF was 2-3 times higher in magnitude $\left(827 \mathrm{mV} \mathrm{cm}^{-1} \mathrm{Oe}^{-1}\right)$. (2) Texturing further increases the magnitude of $\alpha_{E} \quad(1346 \mathrm{mV}$ $\mathrm{cm}^{-1} \mathrm{Oe}^{-1}$ ) by $63 \%$. (3) Textured composites show low loss

TABLE I. Dielectric and magnetoelectric properties of co-fired layered ME composite $(1 \mathrm{kHz})$.

\begin{tabular}{lccccc}
\hline \hline Sample & Electrode & $\varepsilon_{\mathrm{r}}$ & $\begin{array}{c}\tan \delta \\
(\%)\end{array}$ & $\begin{array}{c}\mathrm{d} E / \mathrm{d} H(\max ) \\
{\left[\mathrm{mV} \mathrm{cm}^{-1} \mathrm{Oe}^{-1}\right]}\end{array}$ & $\begin{array}{c}\mathrm{d} E / \mathrm{d} H \text { (zero-bias) } \\
{\left[\mathrm{mV} \mathrm{cm}^{-1} \mathrm{Oe}^{-1}\right]}\end{array}$ \\
\hline C-N/R/N & Co-fired & 1336 & 1.1 & 827 & 510 \\
C-N/T/N & Co-fired & 1135 & 0.7 & 1346 & 1214 \\
\hline \hline
\end{tabular}


due to "engineered domain configuration." (4) The dielectric constant of textured sample was suppressed by using low permittivity BT template crystals. (5) Co-fired textured composites show large $\alpha_{E}$ under zero-bias of the order of $1214 \mathrm{mV}$ $\mathrm{cm}^{-1} \mathrm{Oe}^{-1}$. Taking into account Eq. (2) and the results in Table I, the giant ME response of co-fired textured sample can be easily explained. We should also point out that lower losses of textured sample play an important role in enhancing the $\alpha_{E}$ which can be understood by taking into account the modified piezoelectric voltage coefficient $\left(g_{31}\right)$ given as ${ }^{29}$

$$
g_{31}^{*}=g_{31} \frac{1}{\left(\tan \delta^{\prime}+\tan \theta^{\prime}+\frac{C-C_{f}}{C_{f}}\right)},
$$

where $C-C_{f} / C_{f}$ is the rate of capacitance change, $C$ is capacitance at a given frequency, $C_{\mathrm{f}}$ is capacitance at $1 \mathrm{kHz}$, and $\tan \delta^{\prime}$ and $\tan \theta^{\prime}$ are the intensive dielectric loss and piezoelectric loss, respectively.

In conclusion, NCZF/PMN-PT/NCZF layered composite with $\mathrm{Ag}$ inner electrodes were successfully co-fired at low temperature $\left(930^{\circ} \mathrm{C}\right)$. The co-fired NCZF/textured PMN-PT/ NCZF layered composites exhibited $5 \times$ increase in $\alpha_{E}$ compared to type-A structure (Metglas/PMN-PT/Metglas). Further, these composites exhibited giant self-bias phenomenon which was associated with hysteresis of NCZF magnetostrictive materials. These cost-effective composites with excellent ME properties open the possibility of mass production of numerous magnetoelectric applications as highlighted in Ref. 19.

S.P. and Y.Y. would like to acknowledge the financial support from DARPA. Y.Z. was supported through the funding from AFOSR. The authors also thank the Center for Energy Harvesting Materials and Systems (CEHMS) for access to its facilities.

${ }^{1}$ W. Eerenstein, N. D. Mathur, and J. F. Scott, Nature (London) 442, 759 (2006).

${ }^{2}$ N. A. Spaldin and M. Fiebig, Science 309, 391 (2005).
${ }^{3}$ C. W. Nan, M. I. Bichurin, S. X. Dong, D. Viehland, and G. Srinivasan, J. Appl. Phys. 103, 031101 (2008).

${ }^{4}$ G. Srinivasan, Annu. Rev. Mater. Res. 40, 153 (2010).

${ }^{5}$ S. X. Dong, J. Y. Zhai, J. F. Li, and D. Viehland, Appl. Phys. Lett. 89, 252904 (2006).

${ }^{6}$ Y. J. Wang, D. Gray, D. Berry, J. Q. Gao, M. H. Li, J. F. Li, and D. Viehland, Adv. Mater. 23, 4111 (2011).

${ }^{7}$ J. Ma, J. M. Hu, Z. Li, and C. W. Nan, Adv. Mater. 23, 1062 (2011).

${ }^{8}$ R. C. Kambale, D. Y. Jeong, and J. Ryu, Adv. Condens. Matter Phys. 2012, 824643 (2012).

${ }^{9}$ M. H. Li, D. Berry, J. Das, D. Gray, J. F. Li, and D. Viehland, J. Am. Ceram. Soc. 94, 3738 (2011).

${ }^{10}$ C. Israel, N. D. Mathur, and J. F. Scott, Nat. Mater. 7, 93 (2008).

${ }^{11}$ G. Srinivasan, E. T. Rasmussen, J. Gallegos, R. Srinivasan, Y. I. Bokhan, and V. M. Laletin, Phys. Rev. B 64, 214408 (2001).

${ }^{12}$ M. I. Bichurin, V. M. Petrov, and G. Srinivasan, Phys. Rev. B 68, 054402 (2003).

${ }^{13}$ G. L. Messing, S. Trolier-McKinstry, E. M. Sabolsky, C. Duran, S. Kwon, B. Brahmaroutu, P. Park, H. Yilmaz, P. W. Rehrig, K. B. Eitel, E. Suvaci, M. Seabaugh, and K. S. Oh, Crit. Rev. Solid State 29, 45 (2004).

${ }^{14}$ Y. K. Yan, K. H. Cho, and S. Priya, Appl. Phys. Lett. 100, 132908 (2012).

${ }^{15}$ Y. Saito, H. Takao, T. Tani, T. Nonoyama, K. Takatori, T. Homma, T. Nagaya, and M. Nakamura, Nature (London) 432, 84 (2004).

${ }^{16}$ Y. K. Yan, H. P. Zhou, W. Zhao, and D. Liu, J. Electroceram. 21, 246 (2008).

${ }^{17}$ E. M. Sabolsky, S. Trolier-McKinstry, and G. L. Messing, J. Appl. Phys. 93, 4072 (2003).

${ }^{18}$ Y. Yan, Y. U. Wang, and S. Priya, Appl. Phys. Lett. 100, 192905 (2012).

${ }^{19}$ See supplementary material at http://dx.doi.org/10.1063/1.4791685 for experimental procedure and property of different type of layered ME composites.

${ }^{20}$ Y. K. Yan, K. H. Cho, and S. Priya, J. Am. Ceram. Soc. 94(6), 1784 (2011).

${ }^{21}$ C. S. Park and S. Priya, J. Am. Ceram. Soc. 94, 1087 (2011).

${ }^{22}$ S. E. Park and T. R. Shrout, J. Appl. Phys. 82, 1804 (1997).

${ }^{23}$ H. X. Fu and R. E. Cohen, Nature (London) 403, 281 (2000).

${ }^{24}$ S. C. Yang, C.-S. Park, K.-H. Cho, and S. Priya, J. Appl. Phys. 108, 093706 (2010).

${ }^{25}$ Y. Zhou, S. C. Yang, D. J. Apo, D. Maurya, and S. Priya, Appl. Phys. Lett. 101, 232905 (2012).

${ }^{26}$ U. Laletin, G. Sreenivasulu, V. M. Petrov, T. Garg, A. R. Kulkarni, N. Venkataramani, and G. Srinivasan, Phys. Rev. B 85, 104404 (2012).

${ }^{27}$ Y. Chen, S. M. Gillette, T. Fitchorov, L. Jiang, H. Hao, J. Li, X. Gao, A. Geiler, C. Vittoria, and V. G. Harris, Appl. Phys. Lett. 99, 042505 (2011).

${ }^{28}$ V. K. Vlasko-Vlasov, Y. K. Lin, D. J. Miller, U. Welp, G. W. Crabtree, and V. I. Nikitenko, Phys. Rev. Lett. 84, 2239 (2000).

${ }^{29}$ K. H. Cho, C. S. Park, and S. Priya, Appl. Phys. Lett. 97, 182902 (2010). 